\title{
High-density tissue microarrays from prostate needle biopsies
}

\author{
F McCarthy, ${ }^{1}$ N Dennis, ${ }^{1}$ P Flohr, ${ }^{1}$ S Jhavar, ${ }^{2}$ C Parker, ${ }^{2}$ C S Cooper ${ }^{1}$
}

${ }^{1}$ Institute of Cancer Research, Male Urological Cancer Research Centre, Sutton, UK ${ }^{2}$ The Royal Marsden NHS Foundation Trust, Sutton, UK

\section{Correspondence to}

Professor C Cooper, Institute of Cancer Research, Male Urological Cancer Research Centre, 15 Cotswold Road, Sutton, Surrey SM2 5NG, UK; colin.cooper@icr.ac.uk

Accepted 3 September 2010 Published Online First 15 November 2010

\section{ABSTRACT}

Background Formalin-fixed prostate biopsies are frequently the only tissue collected at the time of prostate cancer diagnosis. There is therefore a requirement for techniques that allow the use of these prostate biopsy specimens in a high-throughput analysis of immunohistochemical and fluorescence-in-situhybridisation-detected biomarkers.

Methods The authors have previously described methods that allow tissue microarray (TMA) construction from prostate biopsies. Here, we describe significant technical innovations that provide an easier and more robust system of biopsy-TMA construction.

Results and discussion The TMAs produced are of a high density (up to 104 cores each, $8 \times 13$ ) and allow a multiplex analysis of biomarkers in the context of clinical trials.

\section{BACKGROUND}

Although prostate cancer is a common disease (over 500000 cases are diagnosed in North America and Europe each year), there is considerable variation in its natural history. ${ }^{1}$ Many cancers are indolent and will never become life threatening while other cases, with an initially similar appearance, may rapidly become life threatening. Unfortunately, established prognostic factors (Gleason score, $\mathrm{T}$ stage, blood prostate-specific antigen, cancer volume) are inadequate, and it is important to identify new biomarkers that can predict more precisely the clinical behaviour of individual patients. We have previously reported methods that can be used to test biomarkers in formalin-fixed needle biopsies taken from patients with prostate cancer at the time of diagnosis. In each method, we reorientate the biopsies into a vertical orientation allowing construction of biopsy TMAs and subsequent analysis of the markers in cross-sections taken from each biopsy TMA. In our original technique, a maximum of 20 biopsy specimens could be included within each TMA. ${ }^{2}$ A particular problem with this method is that the cutting out of small regular 'checkers' of wax containing a segment of the biopsy on one face, which is required for the construction of the biopsy TMA, is technically demanding and time-consuming. To address this problem, we recently presented a simplified technique for cutting wax checkers that allowed construction of biopsy TMAs containing 54-72 checkers. ${ }^{3}$ The technique can be used to examine both IHC and FISH based biomarkers. ${ }^{3}$ These approaches have been validated in studies of the biomarker Ki67 in biopsy specimens from active surveillance patients, ${ }^{4}$ for the biomarkers Hif- $1 \alpha$, VEGF, osteopontin, MDM2, p53 and $\mathrm{Bcl}-2$ in biopsy specimens from radiotherapy patients. ${ }^{5}{ }^{6}$ Here, we present substantial improvements of this procedure that allows more rapid construction of TMAs containing up to 104 biopsy cores.

\section{TECHNICAL DEVELOPMENT}

Prostate biopsy samples for these studies were collected from men with untreated, localised (clinical stage $\mathrm{T} 1 / 2 \mathrm{a}$, Gleason score $\leq 3+4$; prostatespecific antigen $<15 ; \leq 50 \%$ positive cores) prostate cancer who were managed in a prospective active surveillance study at the Royal Marsden Hospital NHS Foundation Trust. The methods for selecting samples for biopsy TMA construction from patients with prostate cancer entered into active surveillance have been described previously. ${ }^{4}$ All patients gave their written consent to take part in the active surveillance study, which was approved by the local research ethics committee.

The new procedure consists of two steps. In the first step, biopsy donor blocks were constructed using previously reported methods, ${ }^{2}$ but with only five biopsy checkers per block (figure 1A-F). The height of the 'checker' was specifically kept at around $4 \mathrm{~mm}$ (figure $1 \mathrm{~A}, \mathrm{~B}$ ) as previously reported. ${ }^{3}$ A variation in the new procedure was that the end of the core was painted with a red dot to identify the core end after embedding (figure 1C), and the side of the checker opposing the biopsy core was painted blue (figure 1D) for orientation. A finished biopsy 'checker' (figure 1E) and donor block (after paraffin wax embedding) (figure 1F) are shown.

In the second step, we use the conventional TMA construction techniques described by Kononen $e t a l^{7}$ to punch out the needle biopsies from the donor block and reset them in a recipient wax block. A hollow needle punch was pushed into the donor block using an MTA1 Manual Tissue Arrayer (Beecher Instruments, Silver Spring, Maryland), to produce a $1.5 \mathrm{~mm}$ diameter core with $4 \mathrm{~mm}$ depth (figure 2A) that encompassed a biopsy specimen: the red marker dot (figure $1 \mathrm{~F}$ ) was used in each case to define the position that was punched. Each donor core was then transferred to a $1.5 \mathrm{~mm}$ diameter, $4 \mathrm{~mm}$ deep hole that had been punched into a recipient wax block (figure $2 \mathrm{~B}$ ). In total, a maximum of 104 cores $(8 \times 13)$ could be arrayed in each recipient block. Usually a number of blank spaces were left in each recipient block defining a unique pattern for block identification. This transfer procedure was usually highly efficient: a detailed examination of the many of the donor blocks following transfer failed to identify residual tissue. The only problems encountered were with very curved biopsies specimens that were not 

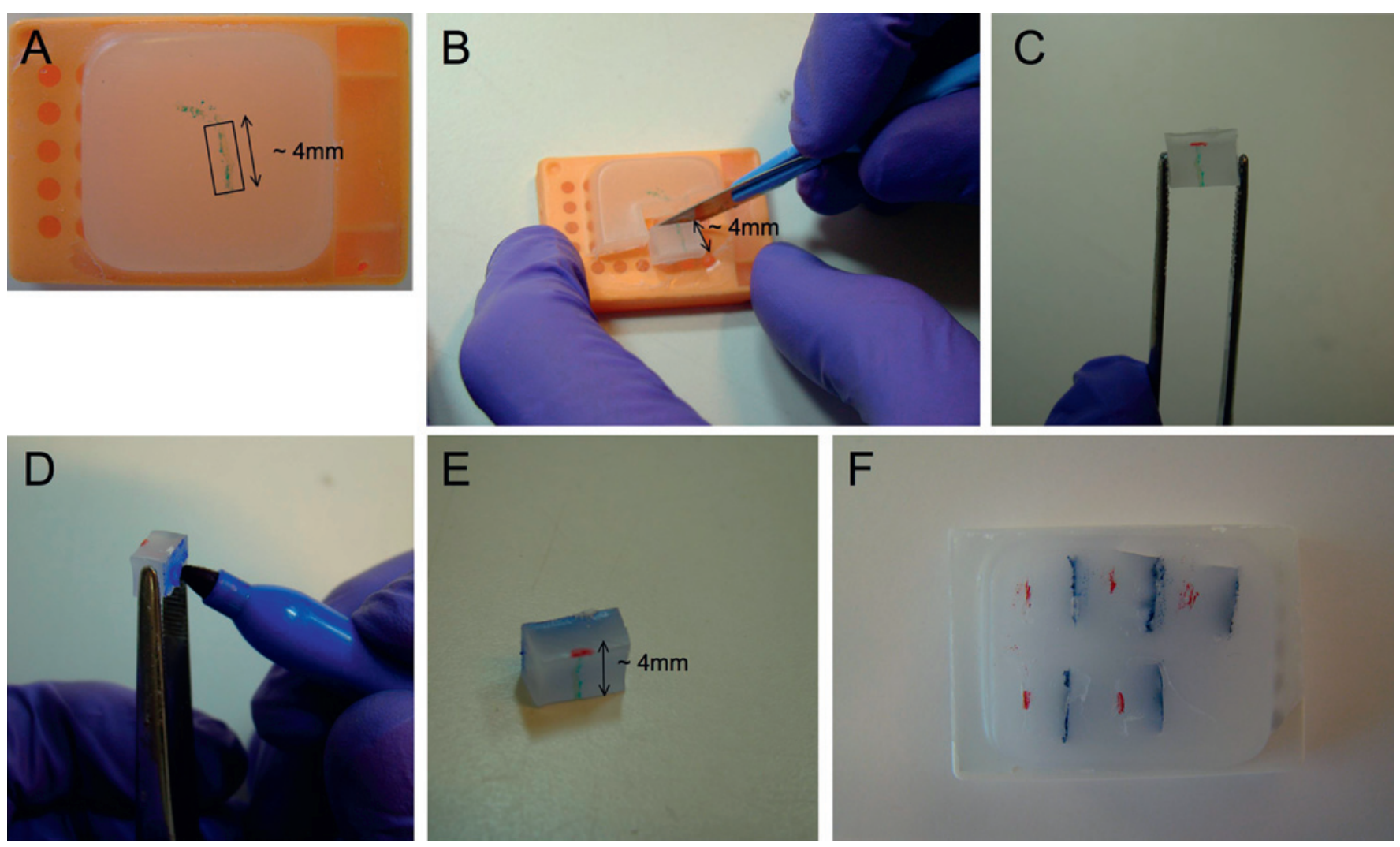

Figure 1 Construction of biopsy donor blocks. For clarity, the formalin-fixed prostate cancer needle biopsy has been coloured green. A malignant portion of the biopsy was marked and represented here by a black rectangle (A). The biopsy was then cut from its original block with a longitudinal length of about $4 \mathrm{~mm}$ to create a 'checker' (B). The cancerous end of the biopsy 'checker' was coloured red (C), and the opposing side to the biopsy was coloured blue (D) for orientation. A finished checker (E) and donor block after embedding (F) are pictured.

Figure 2 Biopsy tissue microarray (TMA) construction. An MTA1 manual tissue arrayer was used to punch out a biopsy core from a donor block (A). The biopsy core was then transferred to a recipient wax block (B). (C) Example of a finished biopsy TMA with (D) corresponding H\&E. A black cross denotes the position of blank spaces.
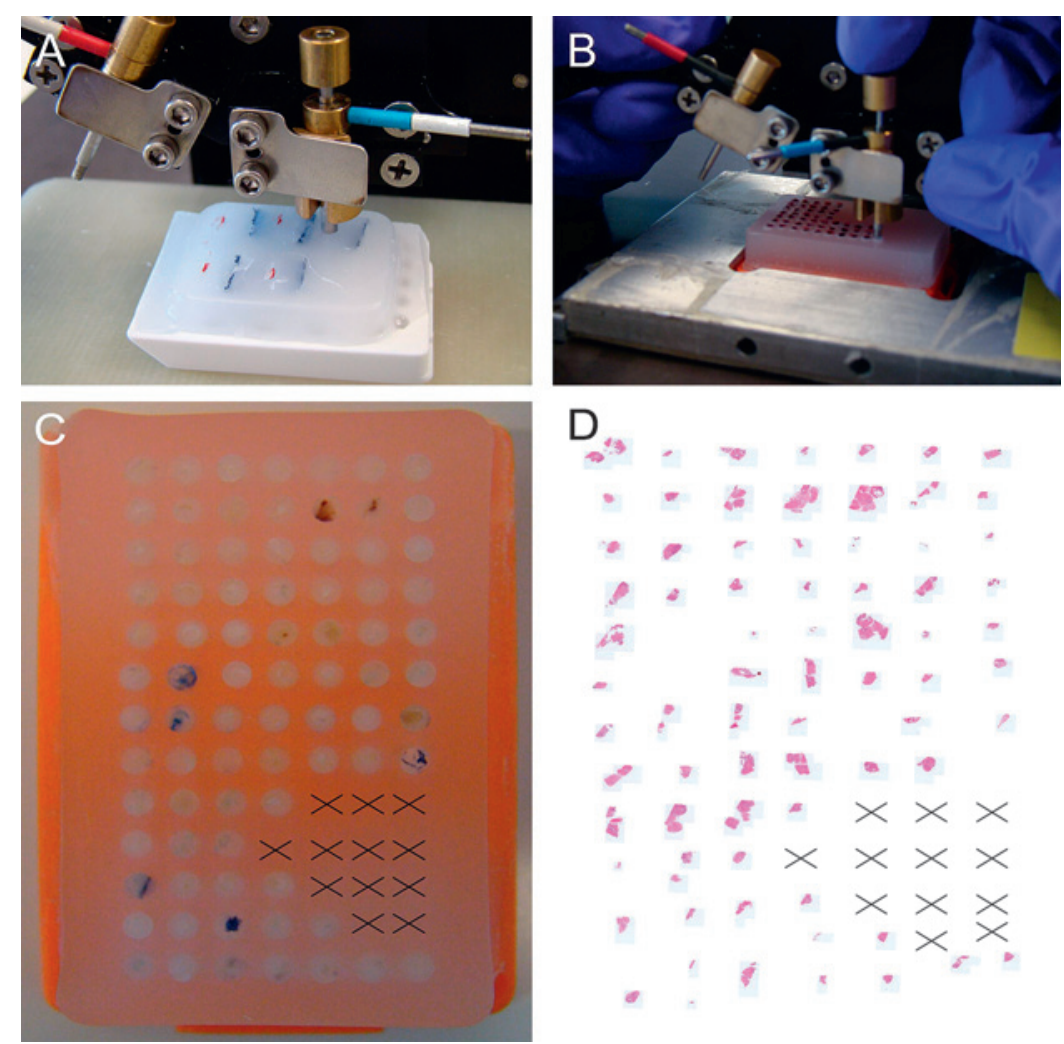


\section{Take-home messages}

- There is an urgent need to identify new biomarkers that will aid in improved targeting of radical treatments in patients with prostate cancer.

- To identify such biomarkers, it is essential to perform tests on samples taken from the patient at the time of diagnosis, which usually only include blood, urine and transultrasound-guided prostate needle biopsy samples.

- A novel method is presented that allows the production of high-density tissue microarrays from needle biopsies taken from the prostate at the time of cancer diagnosis.

- The biopsy TMAs produced can be used for the multiplex analysis of potential biomarkers detected by immunohistochemistry or by fluorescence in situ hybridisation in prostate needle biopsies taken from patients entered into clinical trials.

suitable for arraying using this procedure. Preparation and sectioning of biopsy TMA blocks were carried out exactly as described previously. ${ }^{2}$ An example biopsy TMA block constructed by this method is shown in figure $2 \mathrm{C}$, and the corresponding $\mathrm{H} \& \mathrm{E}$ section is shown in figure $2 \mathrm{D}$. The method was initially used to construct biopsy TMAs in a $13 \times 8$ format (104 cores), but this created brittle block edges, and we consider the $91(13 \times 7)$ pattern to represent the most robust format. We have now used this procedure to construct 12 TMAs from around 1000 biopsies selected from Active Surveillance and MRC RT01 Radiotherapy trials ${ }^{4-6}$ including the coring and resetting of all biopsies in low-density TMAs constructed using our original method. ${ }^{2}$

\section{DISCUSSION}

If biomarkers are to be used clinically for stratifying prostate cancer, they must be tested and validated in tissue obtained from the patient at the time of diagnosis, which usually just includes transrectal-ultrasound-guided prostate needle biopsy, blood or urine. Examination of the prostate biopsy samples yields valuable information including Gleason grade and extent of disease, which facilitate decision-making on the appropriate treatment. The platform that we have developed additionally allows the use of formalin-fixed needle biopsies in multiplex analysis of biomarkers in the precise setting in which they would be used clinically. The multiplex analysis of biomarkers is critical because it is probable that a combination of biomarkers, rather than a single biomarker, will provide the best prognostic or diagnostic information. ${ }^{8}$ Needle biopsies are also taken at the time of diagnosis from many other human malignancies, including oral cancer, breast cancer and lymph-node metastases, so the techniques developed here for prostate cancer may also have relevance to other diseases. ${ }^{9-11}$ In conclusion, the improved strategy for biopsy-TMA construction reported here represents a rapid and robust method for producing high-density biopsy-TMA construction that can be used to assess biomarkers in the context of clinical trials.

Acknowledgements We thank $C$ Hughes for typing the manuscipt.

Funding The work was funded by the National Cancer Research Institute, The Grand Charity of Freemasons, The Rosetrees Trust, and The Prostate Cancer Charity.

Competing interests None.

Ethical approval Ethics approval was provided by The Royal Marsden Research Ethics Committee.

Provenance and peer review Not commissioned; externally peer reviewed.

\section{REFERENCES}

1. Jemal A, Siegel R, Ward E, et al. Cancer statistics, 2006. CA Cancer J Clin 2006; 56:106-30

2. Jhavar S, Corbishley CM, Dearnaley D, et al. Construction of tissue microarrays from prostate needle biopsy specimens. Br J Cancer 2005;93:478-82.

3. McCarthy F, Fletcher A, Dennis N, et al. An improved method for constructing tissue microarrays from prostate needle biopsy specimens. J Clin Pathol 2009:62:694-8.

4. Jhavar S, Bartlett J, Kovacs G, et al. Biopsy tissue microarray study of Ki-67 expression in untreated, localized prostate cancer managed by active surveillance. Prostate Cancer Prostatic Dis 2009;12:143-7.

5. Vergis R, Corbishley C, Norman A, et al. Intrinsic markers of tumour hypoxia and angiogenesis in localised prostate cancer predict the outcome of radical treatment Lancet Oncol 2008:9:342-51.

6. Vergis R, Corbishley CM, Thomas $\mathrm{K}$, et al. Expression of Bcl-2, p53, and MDM2 in localized prostate cancer with respect to the outcome of radical radiotherapy dose escalation. Int J Radiat Oncol Biol Phys 2010;78:35-41.

7. Kononen J, Bubendorf L, Kallioniemi A, et al. Tissue microarrays for high-throughput molecular profiling of tumor specimens. Nat Med 1998;4:844-7.

8. Tricoli JV, Schoenfeldt M, Conley BA. Detection of prostate cancer and predicting progression: current and future diagnostic markers. Clin Cancer Res 2004:10:3943-53.

9. Jereczek-Fossa BA, Jassem J, Orecchia R. Cervical lymph node metastases of squamous cell carcinoma from an unknown primary. Cancer Treat Rev 2004;30:153-64.

10. Morrow M, Strom EA, Bassett LW, et al. Standard for breast conservation therapy in the management of invasive breast carcinoma. CA Cancer J Clin 2002; 52:277-300

11. Neville BW, Day TA. Oral cancer and precancerous lesions. CA Cancer J Clin 2002; 52:195-215. 